\title{
Roadway Condition Survey Technologies: Past, Presence and Trend
}

\author{
Runhua Guo* \\ Department of Civil Engineering, Tsinghua University, China
}

*Corresponding author: Runhua Guo, Department of Civil Engineering, Tsinghua University, China.

Received Date: April 27, 2019

Published Date: May 07, 2019

\begin{abstract}
Modern highway infrastructure management is deeply involved with a data-driven process, both at project-level and network-level. The vast amount of data come from the roadway conditions. The roadway condition survey technologies have evolved from manual to automated. First, the survey technologies for roadway conditions in the past are reviewed. Second, the on-going popular 3-D technology is highlighted. Third, this article sheds lights on the future trend in the automated survey area involving both hardware and software aspects. Last, the relevant specification/ protocols involved in this area are briefly discussed.
\end{abstract}

Keywords: Roadway conditions; 3-D Technology; Manual survey; Automated survey

\section{Introduction}

Roadway condition plays a vital role in highway infrastructure performance monitoring and management. It serves as the base for 1) evaluation of existing health status, 2) input for future performance prediction, and 3) decision making and budget allocation in Maintenance and Repair (M\&R) work. All highway agencies rely heavily on the condition data of their road network. The condition survey has been conducted for many decades. The items in roadway condition survey mainly include distress and ride quality [1]. Regarding the distress, it is composed of varying types of cracks, rutting, faulting, pothole, and son on [2]. The ride quality can be expressed by expert rating or International Roughness Index (IRI). In recent years, additional roadway surface characteristics have gained more attention such as texture, skid, noise, and others due to safety and environmental consideration. Due to the vast demand in roadway condition survey, the survey methods and technologies have evolved significantly. In particular, the roadway industry has been experiencing unprecedent progress in the roadway survey technology due to the advance in other technologies in electronics, optics, and computer, etc.

\section{Past Technologies}

There are two approaches in roadway condition survey: manual and automated [3]. The manual method has been used for a long because it is simple and easy to perform. Traditionally, a rater could walk on the road and measure the distresses. For accurate measurement, the rater needs use tools such as a tape/ruler to obtain the dimensions. Obviously, this is very labor-intensive and timeconsuming. It also has the potential safety risk as the rater walks on the road, unless traffic control is provided. This measurement is mostly used for project-level survey. The alternative manual survey is referred to as visual survey, which is faster than the "walking" survey. Usually a rater could record the distresses information while riding a vehicle along the road side at a speed of 20 to 30 $\mathrm{km} / \mathrm{h}$. The visual survey is more efficient than walking survey. But it can only provide less detailed distress information than the walking survey. Furthermore, it may suffer from variations among different raters because it is a subjective measurement process. The visual survey has been applied to both project and network levels. The automated road condition survey started around several decades ago, depending on which technology is involved. It mainly uses different sensors installed on a host vehicle to collect roadway surface information. The earliest was the ride quality data collection equipment, referred to as the high-speed inertial profiler originated from a world-bank study [4]. Nowadays it is widely used in pavement community to collect IRI. While it suits for highspeed traveling condition, the data from inertial profiler at low or varying speed condition such as in the urban environment can be misleading due to the limitation in the accelerometer in the sensor 
system. For rutting, multi-point acoustic sensors were used to collect the road surface elevation information to rutting calculation. The disadvantage of acoustic sensor is that the data quality could be affected by temperature, wind, and vehicle speed. This technology also suffered from low sampling rate. Regarding cracking, which is the most challenging in roadway distress survey, the 2-Dimension (2-D) imaging technology was employed around two decades ago. It significantly improved the data collection efficiency so that the large-scale network-level condition survey was made possible. The shortcoming of the 2-D imaging technology comes from the fact that it could be intervened by the ambient light such as the sunshine. For example, it could falsely report cracks at the tree-shadow areas.

\section{Current Focus}

To address the issues in past technologies, the 3-D imaging technology has been launched and developed rapidly since around a decade ago. The key sensors of a 3-D system include a line-scan laser and a 3-D camera [5]. The laser can be featured as 2048 points $(2 \mathrm{~K})$ or 4096 points $(4 \mathrm{~K})$ covering the entire width of a travel lane. As the host vehicle moves, the $\mathrm{x}, \mathrm{y}$, and $\mathrm{z}$ information on the road surface is captured by the camera and recorded at the pixel level. The triangulation algorithm is used to calculate the 3-D related geometry [6]. It should be noted that due to the desired property of the 3-D technology, it is being used to detect a series of roadway conditions including cracking, rutting, texture, and ride quality. In addition to the former two discussed above, the 3-D system has been used in macro-texture data collection [7], varying-speed ride quality data collection in very recent studies [8]. The vehicle operation speed can be up to $100 \mathrm{~km} / \mathrm{h}$. This makes the system ideal for network-level data collection. More specifically, the automated roadway survey is composed two types. One is semi-automated and the other is fully-automated. They differ in the image processing and reporting. Although data collection process could be automatically completed by the sensors installed on the host vehicle, the following task on image recognition, distress classification and processing, and quality control most likely requires human intervention. This reflects the status quo in the data collection area. The ongoing engineering and research effort in roadway condition survey can be found at the Road Profiler User Group Annual Meeting [9].

\section{Future Directions}

In order to move toward fully-automated roadway condition survey, pioneering effort has been made by using the Artificial Intelligence (AI) technology in distress recognition and processing. For example, the Convolutional Neural Network (CNN) was used to extract the crack information on the distress maps based on a large number of pixel-level training data [10]. In this aspect, AI can be a promising tool toward the fully-automated roadway condition survey technology in the future. It is believed the trend in this area also will include the following. On-line data processing: this requires both fast data processing software with very efficient algorithm and hardware such as high-speed GPU. Higher-resolution and wider range sensors: this can provide sub-millimeter level quality image and accommodate all kinds of roadway condition levels.

\section{Specifications and Protocols}

Last but not least, the roadway survey technology development has been always interacted with the corresponding specifications/ protocols in this area. On the one hand, the emergence of the new technology can lead to the establishment of new specifications/ protocols and its update. On the other hand, the continuouslyimproved specifications/protocols can push the technology more reliable and practically applicable in the engineering work. AASHTO and ASTM have published and periodically updated the specifications and protocols on roadway condition equipment technical requirement, measurement methods, data quality, and so on. Some examples are: ride quality, rutting, cracking, texture etc. $[4,11,12]$.

\section{Conclusion}

Numerous studies have been conducted in the past decades to improve the roadway condition survey efficiency and accuracy. Significant effort has been invested into engineering practice in this area parallelly. Nowadays the roadway survey has entered a new era with focus on 3-D imaging and survey. This technology makes the highway infrastructure management a better data-driven process. It has been approved to greatly enhance the highway infrastructure management toward optimized decision making and resource allocation. In the meantime, the current technology is by no means perfect. There is still plenty of room for improvement both in sensors and software.

\section{Acknowledgment}

None.

\section{Conflict of Interest}

No conflict of interest.

\section{References}

1. FHWA (2016) Highway Performance Monitoring System Field Manual. U.S. Federal Highway Administration.

2. LTPP (2003) Distress Identification Manual for the Long-Term Pavement Performance Program.

3. AASHTO (2015) Pavement Design, Construction, and Management: A Digital Handbook ( $1^{\text {st }}$ edn.). American Association of State Highway and Transportation Officials. Washington DC, USA.

4. ASTM E950 / E950M-09 (2018) Standard Test Method for Measuring the Longitudinal Profile of Traveled Surfaces with an AccelerometerEstablished Inertial Profiling Reference. ASTM International, West Conshohocken, PA, USA.

5. Huang Y, Hempel P, Coppenhaver T (2013) Texas Department of Transportation 3D Transverse Profiling System for High Speed Rut Measurement. ASCE Journal of Infrastructure Systems 19(2).

6. Acosta D, Garcia O, Aponte J (2006) Laser Triangulation for Shape Acquisition in a 3-D Scanner Plus Scan. Electronics, Robotics and Automotive Mechanics Conference 2: 14-19.

7. Hong F, Huang Y (2014) Measurement and Characterization of Asphalt Pavement Surface Macrotexture Using 3D Laser Scanning Technology. Journal of Testing and Evaluation, American Society for Testing and Evaluation 42(4): 881-890.

8. Guo R, Yu Z, Zhou Y (2018) Development and Preliminary Evaluation of a Varying-Speed Road Profiler. Journal of Testing and Evaluation.

9. Road Profiler User Group Annual Meeting. 
10. Cha Y, Choi W, Buyukozturk O (2017) Deep Learning-Based Crack Damage Detection Using Convolutional Neural Networks. ComputerAided Civil and Infrastructure Engineering pp.1-18.

11. AASHTO (2017) Designation, PP-70(14). Standard Practice for Collecting the Transverse Pavement Profile. American Association of State Highway and Transportation Officials, Washington DC, USA
12.ASTM E1845-01(2007). Standard Practice for Calculating Pavement Macrotexture Mean Profile Depth. American Society for Testing and Materials, West Conshohocken, PA, USA. 